\title{
COVID-19 encounters reported to the Canadian Anesthesia Incident Reporting System (CAIRS)
}

\author{
W. Scott Beattie, MD, PhD, FRCPC (1) - Kathryn A. Sparrow, MD, \\ FRCPC, MScHQ
}

Received: 10 January 2021 /Revised: 1 February 2021 / Accepted: 5 February 2021 /Published online: 19 February 2021

(c) Canadian Anesthesiologists' Society 2021

\section{To the Editor,}

The Canadian Anesthesiologists' Society created the Canadian Anesthesia Incident Reporting System (CAIRS), ${ }^{\mathrm{A}}$ which is now available to any practitioner in Canada who may wish to enter an anesthesia-related event. ${ }^{1}$ In March 2020, CAIRS was configured to capture coronavirus disease (COVID-19) related encounters. This communication details all COVID-19-related events reported to CAIRS as of 31 December 2020.

Anesthesiologists in Canada have reported 23 COVID19 encounters. Two of these encounters were elective intubations. Eighty-seven percent of the encounters were reported by specialist anesthesiologists; two encounters were reported by trainees. Over $90 \%$ of intubations took place in the Emergency Department, the operating room, or the intensive care unit (ICU). Personal protective equipment (PPE) was universally available. Nevertheless, negative pressure was unavailable in four cases. The American Society of Anesthesiologists' physical status was IV or greater in $70 \%$ of these patients. The median decade of life of patients' age was 60-70 yr. Thirty percent of patients had a body mass index $>30 \mathrm{~kg} \cdot \mathrm{m}^{-2}$. There were no reports of healthcare workers testing positive for COVID 19 after a patient encounter.

Six of the reported cases (26\%) had a COVID-19 diagnosis confirmed by laboratory testing. The remaining

W. S. Beattie, MD, PhD, FRCPC ( $ه)$

University of Toronto, University Health Network, Toronto, ON,

Canada

e-mail: scott.beattie@uhn.on.ca

\section{K. A. Sparrow, MD, FRCPC, MScHQ}

Discipline of Anesthesia, Memorial University of

Newfoundland, St. John's, NL, Canada reports were of patients with symptoms leading to a presumptive COVID-19 diagnosis; these were treated using a COVID-19 intubation protocol but lacked a confirmatory diagnostic test.

In the following, we highlight five scenarios uploaded to the CAIRS COVID-19 website. Two of these illustrate issues associated with institutional COVID-19 protocols; three report situations involving a patient death.

\section{Scenario 1}

A patient in the eighth decade of life underwent an urgent oncologic procedure. The COVID-19 status was unknown but according to COVID-19 protocols, all personnel required airborne PPE. During the procedure, the patient experienced rapid blood loss and prolonged hypotension with systolic blood pressures between 70 and $80 \mathrm{mmHg}$ and a mean arterial pressure of $50 \mathrm{mmHg}$ sustained for more than 30 min. Resuscitation included the administration of crystalloid, blood products, and vasopressors. Postoperative investigations showed an elevated troponin. Prophylactic long-term cardiac care was initiated. The reporting physician felt that, along with poor communication with the surgical team, the PPE hindered the ability to hear the suction and recognize the rapid blood loss.

\footnotetext{
A Canadian Anesthesia Incident Reporting System. Available from URL: https://cairs.ca/ (accessed February 2021).
} 


\section{Scenario 2}

An anesthesiologist working in a tertiary referral centre reported an ongoing concern with COVID-19 protocols. The patient referral base for this institution was described to include a large number of patients with communityacquired COVID-19. However, the receiving hospital was reported to have insufficient resources or testing facilities to screen patients being admitted semi-electively for oncologic surgery. The submitting anesthesiologist expressed concern that the high prevalence of asymptomatic and/or pre-symptomatic carriers places both the surgical patients and healthcare workers at risk and called for systemic COVID-19 testing to safely carry out the provision of care during this pandemic.

\section{Scenario 3}

A patient in the fourth decade of life presented to a rural hospital with tachypnea and signs of worsening respiratory failure suggestive of COVID-19. The patient was known to have a cardiomyopathy and an ejection fraction of $18 \%$. The patient was intubated emergently in the emergency department with the use of ketamine and rocuronium. The patient sustained a cardiac arrest on induction and could not be resuscitated. Post-mortem, a COVID-19 test came back as negative.

\section{Scenario 4}

A patient in the ninth decade of life with a history of hypertension, hypothyroidism, and depression was admitted with a bowel obstruction due to an incarcerated umbilical hernia and presented for emergency surgery. Significant previous contact with a COVID-19-positive patient was noted. A nasopharyngeal swab had been sent; however, the results were not yet available. A nasogastric tube could not be successfully placed due to the patient's uncooperative nature. Prior to induction of anesthesia, COVID-19 operating room protocols and appropriate measures were executed. On induction, the patient regurgitated a large amount of bilious fluid. The oropharynx was immediately suctioned and the patient intubated with a videolaryngoscope. Initially, ventilation and oxygenation were normal. However, towards the end of the case, the patient exhibited increasing oxygen requirements and was transferred intubated and ventilated to the ICU postoperatively. Over the course of the next few days, the patient remained on a high fraction of inspired oxygen $\left(\mathrm{F}_{\mathrm{I}} \mathrm{O}_{2}\right)$, mechanical ventilation, and vasopressors. After five days without improvement, a decision to change the therapeutic goal to comfort care was made.

\section{Scenario 5}

A patient in the sixth decade of life who was under investigation for COVID-19 presented with tachypnea, increasing oxygen requirements, bibasilar crackles, and a chest radiograph showing bibasilar consolidation. The patient was noted to have significant abdominal distension, and abdominal imaging showed distension of the esophagus, stomach, and small bowel. Prominent loops of small bowel were thought to be due to ileus rather than obstruction. The patient developed profound sepsis and lactic acidosis and was brought to the operating room for an emergency laparotomy for perforated viscous. A rapid sequence intubation was performed as per the institutional COVID-19 protocol. At time of laryngoscopy, vomit was noted in the hypopharynx which was suctioned; the patient was then intubated successfully on the first attempt. Transient oxygen desaturation occurred but oxygenation recovered with recruitment maneuvers and controlled ventilation. The endotracheal tube was suctioned but no gastric fluid returned. The stomach returned a large amount of fluid when decompressed with an orogastric tube. Over the course of the surgery, the patient became progressively hemodynamically unstable, requiring triple vasopressor therapy. Oxygen saturation was in the low $90 \mathrm{~s}$ on an $\mathrm{F}_{\mathrm{I}} \mathrm{O}_{2}$ of $0.75 \%$. Postoperatively, the patient was transferred to the ICU but remained unstable requiring multiple vasopressors and a high $\mathrm{F}_{\mathrm{I}} \mathrm{O}_{2}$ on mechanical ventilation to maintain adequate oxygenation, and subsequently died of septic shock.

The above selected cases and narratives highlight the multiple factors that may have contributed to the patient safety incidents submitted to CAIRS. Their analysis presents an opportunity for growth and learning. Breakdowns in processes of care, such as delayed access to rapid COVID-19 testing (scenarios 1-5), communication challenges associated with PPE (scenario 1), alterations in airway management plans and decision-making frameworks based on COVID-19 protocols (scenarios 3, 4 , and 5), and uncertain or undifferentiated clinical issues at the time of care (scenario 3) may have contributed to patient outcomes. In this limited series, one factor stands out, however: $75 \%$ the patients did not have an established COVID-19 diagnosis at the time of anesthetic or airway management. We therefore submit that expanded or rapidly 
available COVID-19 testing results in the perioperative setting could improve patient safety.

The decision to report an incident to CAIRS is a personal choice. Likewise, the level of detail included in a narrative is up to the reporter. We would like to take this opportunity to thank anesthesiologists for reporting events to CAIRS and encourage all Canadian anesthesia care providers to use CAIRS when they experience or witness any adverse event.

Disclosures None.

Funding statement Funded by the Canadian Anesthesiologists' Society.
Editorial responsibility This submission was handled by Dr. Stephan K.W. Schwarz, Editor-in-Chief, Canadian Journal of Anesthesia.

\section{References}

1. Beattie WS, Culwick MD, Grocott HP. Canadian Anesthesia Incident Reporting System (CAIRS): The Canadian Anesthesiologists' Society's National Patient Safety Initiative. Can J Anesth 2018; 65: 749-56.

Publisher's Note Springer Nature remains neutral with regard to jurisdictional claims in published maps and institutional affiliations. 